\title{
The sizes of absorption line systems
}

\author{
Patrick Petitjean ${ }^{1}$ \\ ${ }^{1}$ Institut d'Astrophysique de Paris, 98bis Boulevard Arago, 75014 Paris, France \\ email: petitjean@iap.fr
}

\begin{abstract}
For a long time it has been usual to search for the objects that host the gas from which absorption line systems arise. However, the spatial distribution of metals around galaxies and more generally in the intergalactic medium is complex, and it is often very difficult to associate one absorption system with one galaxy. Except for possibly in the special case of DLA systems, it may be more appropriate to discuss the clustering properties of different classes of objects and to measure their correlation functions. I illustrate this approach with three examples: the distribution of metals around galaxies at intermediate redshift, the modelling of the clustering of $\mathrm{C}$ IV systems, and the determination of the transverse correlation function of the Lyman- $\alpha$ forest.
\end{abstract}

\section{Galactic winds}

Very recently it was realised that galaxies could be associated with powerful winds that can expel a non-negligible amount of metals into the intergalactic medium. Several lines of argument seem to indicate that this is indeed the case: (i) it has been shown that $\mathrm{C}$ IV and $\mathrm{O}$ VI are present in the intergalactic medium down to $\mathrm{H}$ I optical depths as low as $\tau \sim 1$, corresponding to metallicities of about $[\mathrm{C} / \mathrm{H}] \sim-3.2$ (Schaye et al. 2003; Aracil et al. 2004); (ii) it seems that, at least in some cases, the intergalactic medium contains less neutral hydrogen than the global average in the vicinity $\left(\sim 0.5 h^{-1} \mathrm{Mpc}\right)$ of Lyman break galaxies at high redshift (Adelberger et al. 2003), which may indicate that supernova-driven winds from these galaxies are able to drive away the intergalactic gas; (iii) star-burst galaxies at low redshift seem to show evidence of winds with velocities of the order of several hundreds of $\mathrm{km} \mathrm{s}^{-1}$ (Heckman et al. 2000).

Although these arguments are suggestive, the overall picture is far from being fully convincing. The exact mechanisms powering these winds are not known and the fate of the gas is unclear (Mac Low \& Ferrara 1999). Although winds may occur more easily in dwarf galaxies, the metals ejected by massive galaxies may dominate the total budget. In any case the spatial distribution of metals around galaxies must be highly complex. It is therefore not a trivial problem to derive this distribution, and, in the end, to assess which galaxies are responsible for the enrichment of the IGM and what is the impact of these winds on galaxy formation and evolution.

One way to do so is to study the correlation between galaxies and the corresponding absorption line systems detected in the spectrum of background quasars. Indeed this is one of the best ways to correlate the properties of the galaxy itself (star-formation rate, metallicity, morphology) with those of the gas (ionisation state, metallicities, and kinematics). 


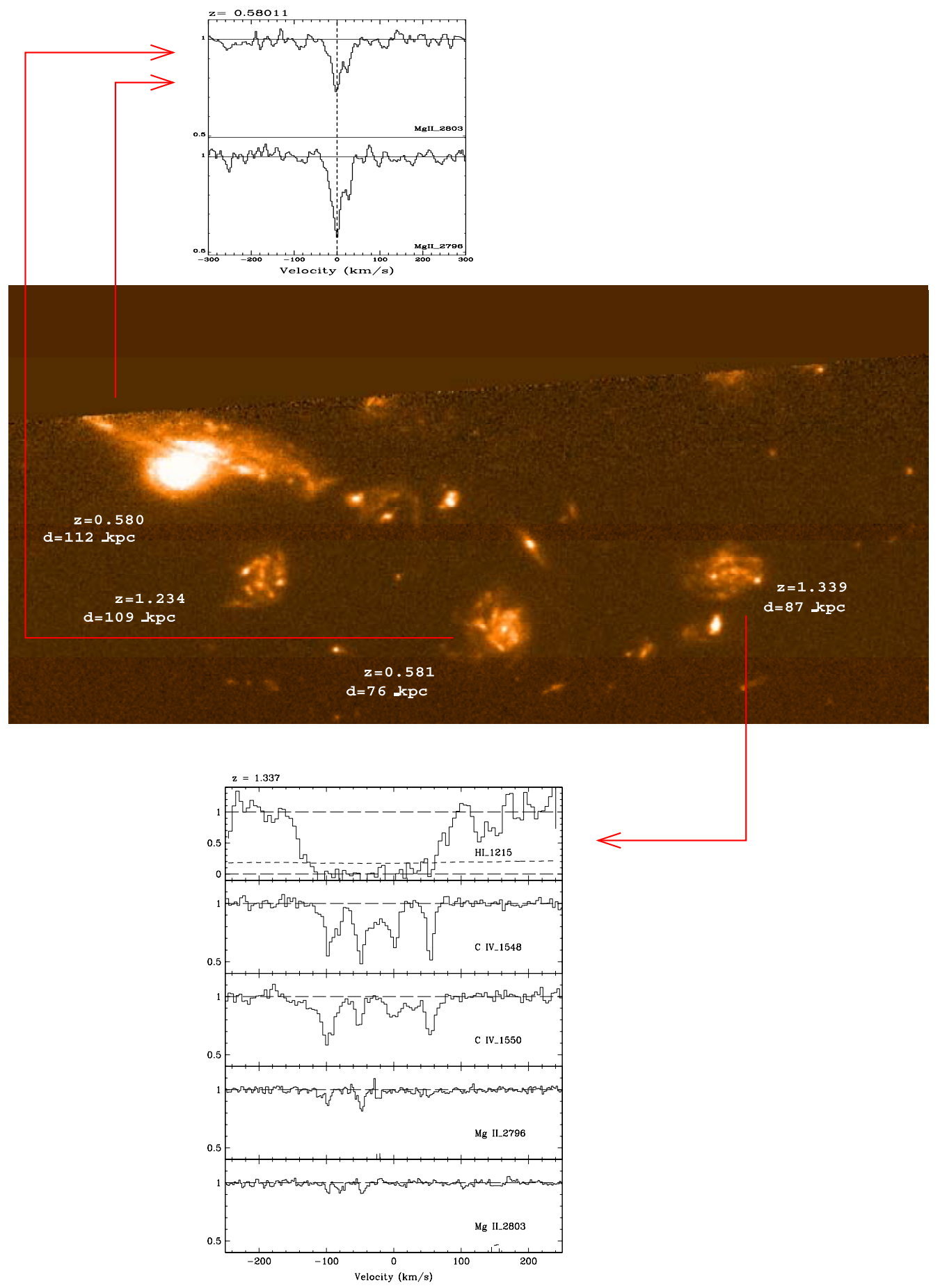

Figure 1. Identification of absorbers in the STIS-HDFS field. The quasar is located about 25 arc-sec South of the image. Several weak Mg II systems are observed at the same redshift as the galaxies. The impact parameters are larger than $100 \mathrm{kpc}$ at $z \sim 0.7$. 


\subsection{The population of $M g$ II absorbing galaxies}

It is well known that at intermediate redshift $\langle z\rangle \sim 0.5-1.0$, strong Mg II systems with equivalent width $W_{\mathrm{r}}(2796) \geqslant 0.3 \AA$ arise in large $R \sim 35 h_{50}^{-1} \mathrm{kpc}$ gaseous halos of a population of bright and fairly blue galaxies with $L>0.3 L^{*}$.

The absorbing galaxies are generally of normal morphology identifiable along the Hubble sequence (Bergeron \& Boissé 1991; Steidel et al. 1997). However, (i) it is very difficult to establish (either by kinematics or by looking at fainter objects in the field) the exact link between the gas and the bright nearby galaxy; (ii) the corresponding Mg II absorptions are quite strong in these studies whereas we know now that the Mg II population is dominated by weak systems.

\subsection{Properties of $M g$ II absorption systems}

The co-moving redshift path density of $\mathrm{Mg}$ II absorbers having $W_{\mathrm{r}}(2796)>0.3 \AA(\mathrm{d} N /$ $\mathrm{d} z \sim 1.2$ at $z \sim 1$ ) is consistent with a non-evolving population of objects. Their number density is similar to the number density of Lyman limit systems over a large redshift range $(0.2<z<2)$. It has therefore been suggested that they arise in optically thick clouds which are part of galactic halos that are not strongly evolving with time. At larger equivalent width, the evolution is much stronger (e.g. Petitjean \& Bergeron 1990; Steidel \& Sargent 1992). The statistical analysis of these observations suggests that the weak systems could be associated with a diffuse environment (e.g. external parts of halos that are at the transition limit with the overall intergalactic medium) that does not evolve much and stays in place over the complete redshift range, whereas the strong systems are directly related to the central regions of the halos that collapse slowly.

High resolution and high $\mathrm{S} / \mathrm{N}$ ratio spectra of quasars give unique information on the kinematics, ionisation state, and chemical composition of the gas in the halos of galaxies over the redshift range $0.2<z<2.2$. Keck observations of Mg II systems at $z \sim 0.7$ with identified absorbing galaxies, have shown that the kinematics and the chemical/ionisation properties of the absorption systems are not correlated with the impact parameter (Churchill et al. 2000). This suggests that the structure of the gas around galaxies is highly perturbed and that big gaseous halos are probably maintained by the energy expelled into the halo by star formation activity in the disk.

\subsection{The weak $M g$ II absorption systems}

The advent of $8-10 \mathrm{~m}$ class telescopes has allowed the detection of a population of weak $\mathrm{Mg}$ II absorbers $\left(0.02<W_{\mathrm{r}}(2796)<0.3 \AA\right)$. The number of absorbers per unit redshift, $d N / d z$, increases as the equivalent width threshold decreases $(\mathrm{d} N / \mathrm{d} z=3$ for $W_{\mathrm{r}}(2796)>20 \mathrm{~m} \AA$ and $=1.2$ for $W_{\mathrm{r}}(2796)>0.3 \AA$ at $\left.\langle z\rangle=0.9\right)$. Therefore at this redshift, the weak $\mathrm{Mg}$ II systems comprise at least $\sim 65 \%$ of the total $\mathrm{Mg}$ II absorption population and about $5 \%$ of the Lyman- $\alpha$ forest lines with $W_{\mathrm{r}}(\operatorname{Ly} \alpha) \geqslant 0.1 \AA$. Other properties point to a different nature for the majority of weak Mg II absorbers with respect to the strong ones: they arise in optically thin neutral hydrogen environments (Churchill et al. 2000); and they are single clouds, in contrast to the complex kinematics of strong $\mathrm{Mg}$ II absorbers (Rigby et al. 2002).

In the few cases for which imaging is available, it is unclear whether they are associated with luminous counterparts or not (at least up to $\sim 100 h_{50}^{-1} \mathrm{kpc}$; see e.g. Fig. 1). Assuming that all Mg II absorbers arise in luminous galaxies would imply spherical halos of radius larger than $R \sim 125 h_{50}^{-1} \mathrm{kpc}$. If true, it is probable that part of the gas located that far from the central regions is no longer bound to the galaxy. They could therefore also trace sheets or filaments from the large scale structure network. It is therefore crucial for our understanding of the distribution of gas in halos of galaxies that this latter point 

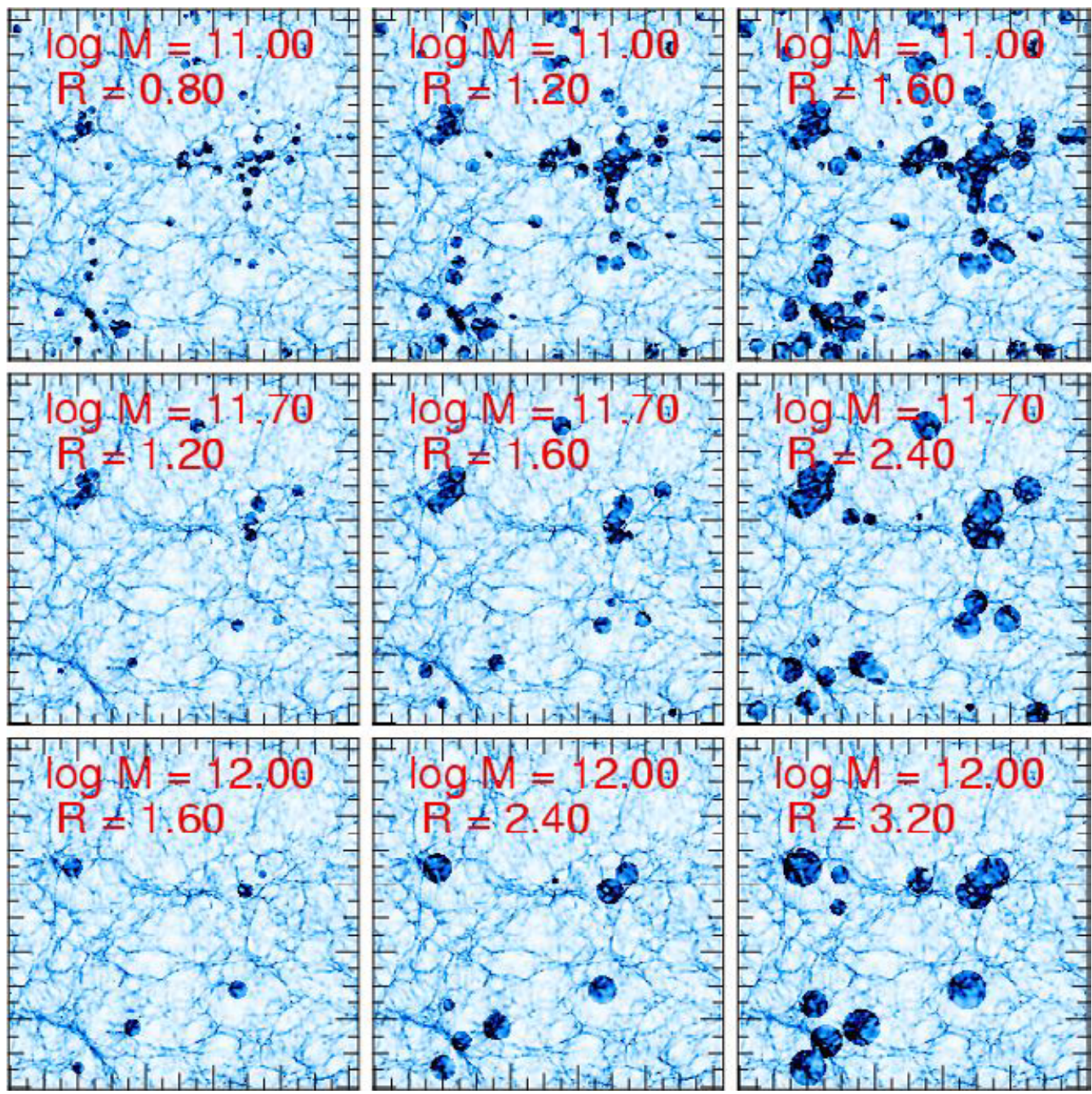

Figure 2. The C IV distribution from a $z=3$ slice in a hydrodynamical simulation meant to reproduce the velocity correlation function and column density distribution of C IV systems. Dark regions are those contained within a distance $R_{\mathrm{s}}$ from a dark matter halo of mass $M_{\mathrm{s}}$, with parameters as labelled in the panels. Preferred models are in the right-side row for which filling factors are $16.1 \%, 16.7 \%$, and $11.6 \%$ from top to bottom respectively.

be investigated in more detail. In particular it is important to go faint and therefore to investigate the possible associations using photometric redshifts. This is what we plan to do in several fields of the CFHTLS survey.

\section{Correlation of C IV systems}

Whereas Mg II is thought to be associated with optically thick gas, C IV arises in gas of higher ionisation and is expected to be spread to larger distances from the location of star formation activity. Therefore it is even more difficult to discuss the association of $\mathrm{C}$ IV with galaxies (see an example in Fig. 1). One way to investigate this is to compare the observed clustering properties of $\mathrm{C}$ IV absorption lines to the output of $N$-body 
simulations. We have used $619 \mathrm{C}$ IV absorption components with $N \geqslant 10^{12} \mathrm{~cm}^{-2}$ observed in 19 high signal-to-noise (60-100 per pixel), high resolution $(R=45,000)$ quasar spectra. The line-of-sight correlation function $\xi_{\mathrm{CIV}}(v)$ exhibits a steep decline at large separations and a flatter profile below $\sim 150 \mathrm{~km} \mathrm{~s}^{-1}$. These features do not depend on absorber column depths, although there are hints that the overall amplitude of $\xi_{\text {CIV }}$ increases with time over the redshift range 1.5-3 (see Scannapieco et al. 2005). Carrying out a detailed smoothed particle hydrodynamics simulation $\left(2 \times 320^{3}, 57 \mathrm{Mpc}^{3}\right.$ co-moving), we showed that the $\mathrm{C}$ IV correlation function can not be reproduced by models in which the IGM metallicity is a constant or a local function of over-density $\left(Z \propto \Delta^{2 / 3}\right)$. However, the properties of $\xi_{\mathrm{CIV}}(v)$ are consistent with a model in which metals are confined within bubbles with a typical radius $R_{\mathrm{S}}$ about sources of mass $\geqslant M_{\mathrm{s}}$. We derive best-fit values of $R_{\mathrm{S}} \sim 2$ co-moving $\mathrm{Mpc}$ and $M_{\mathrm{s}} \sim 10^{12} \mathrm{M}_{\odot}$ at $z=3$ (see Fig. 2).

\section{Longitudinal and transverse correlation function in the Lyman- $\alpha$ forest}

The intergalactic medium (IGM) is revealed by the numerous H I absorption lines seen in the spectra of distant quasars, the so-called Lyman- $\alpha$ forest. There is a long history of using the flux transverse correlation in the Lyman- $\alpha$ forest spectra of QSO pairs to measure the spatial extent of the corresponding absorbing structures. The Lyman$\alpha$ forests in the spectra of multiple images of lensed quasars with separations of the order of a few arc-sec (e.g. Smette et al. 1995) appears nearly identical implying that the absorbing structures have sizes $>50 h_{75}^{-1} \mathrm{kpc}$. Significant correlation still exists for separations of a few to ten arc-min suggesting a coherence length as large as $500 h_{75}^{-1} \mathrm{kpc}$ (e.g. Petitjean et al. 1998; Crotts \& Fang 1999; Young et al. 2001; Aracil et al. 2001). The transverse correlation function can be used to constrain the spatial distribution of the IGM and, together with the longitudinal correlation function, can lead to constraints on the cosmological parameters (and especially $\Omega_{\Lambda}$ through the Alcock-Paczyńsky test (Alcock \& Paczyński, 1979).

We have used 33 pairs of quasars observed with FORS at the VLT to derive the flux correlation functions of the Lyman- $\alpha$ forest. The latter are defined as in Rollinde et al. (2003):

$$
\xi_{f}(\theta, \Delta v)=\langle(\mathcal{F}(\theta, \lambda+\Delta \lambda)-\langle\mathcal{F}\rangle)(\mathcal{F}(0, \lambda)-\langle\mathcal{F}\rangle)\rangle_{\lambda},
$$

where $\mathcal{F}$ is the normalised flux along two lines of sight with separation $\theta$ at a mean redshift $z ; \Delta \lambda=\lambda_{0}(1+z) \times \Delta v / c$, with $\lambda_{0}=1215.67 \AA$ the hydrogen Lyman- $\alpha$ restwavelength, and $c$ denotes the speed of light. The velocity distance corresponding to the angular separation $\theta$ can be written as $\Delta v_{\perp}=c f \theta$, where $f(z)$ is a scaling factor which depends on the angular diameter distance (see McDonald 2003 and Rollinde et al. 2003). In the following we use $\mathrm{H}_{0}=70 \mathrm{~km} / \mathrm{s} / \mathrm{Mpc}, \Omega_{\mathrm{m}}=0.3$, and $\Omega_{\Lambda}=0.7$. Therefore, at $z=2, \Delta v=100 \mathrm{~km} \mathrm{~s}^{-1}$ corresponds to $\sim 1$ arc-min.

For all correlation functions, the mean flux, $\langle\mathcal{F}\rangle$, is taken as the average over all the pixels along the corresponding line of sight. The effect of changing the definition of $\langle\mathcal{F}\rangle$ is mostly to change the normalisation of the correlation function. Note that the normalisation of the simulated correlation functions is a free parameter. Indeed, the mean flux in the simulation depends on the intensity of the ionising background $\left(J_{21}\right)$. To fix this parameter we adjust the flux PDF in the simulation to the observed one. One quasar pair yields one data point of the transverse correlation function [corresponding to $\Delta v=0$ at the transverse separation $\left.\theta_{\mathrm{i}}, \chi\left(\theta_{\mathrm{i}}\right) \equiv \xi_{f}\left(\theta_{\mathrm{i}}, 0\right)\right]$. 


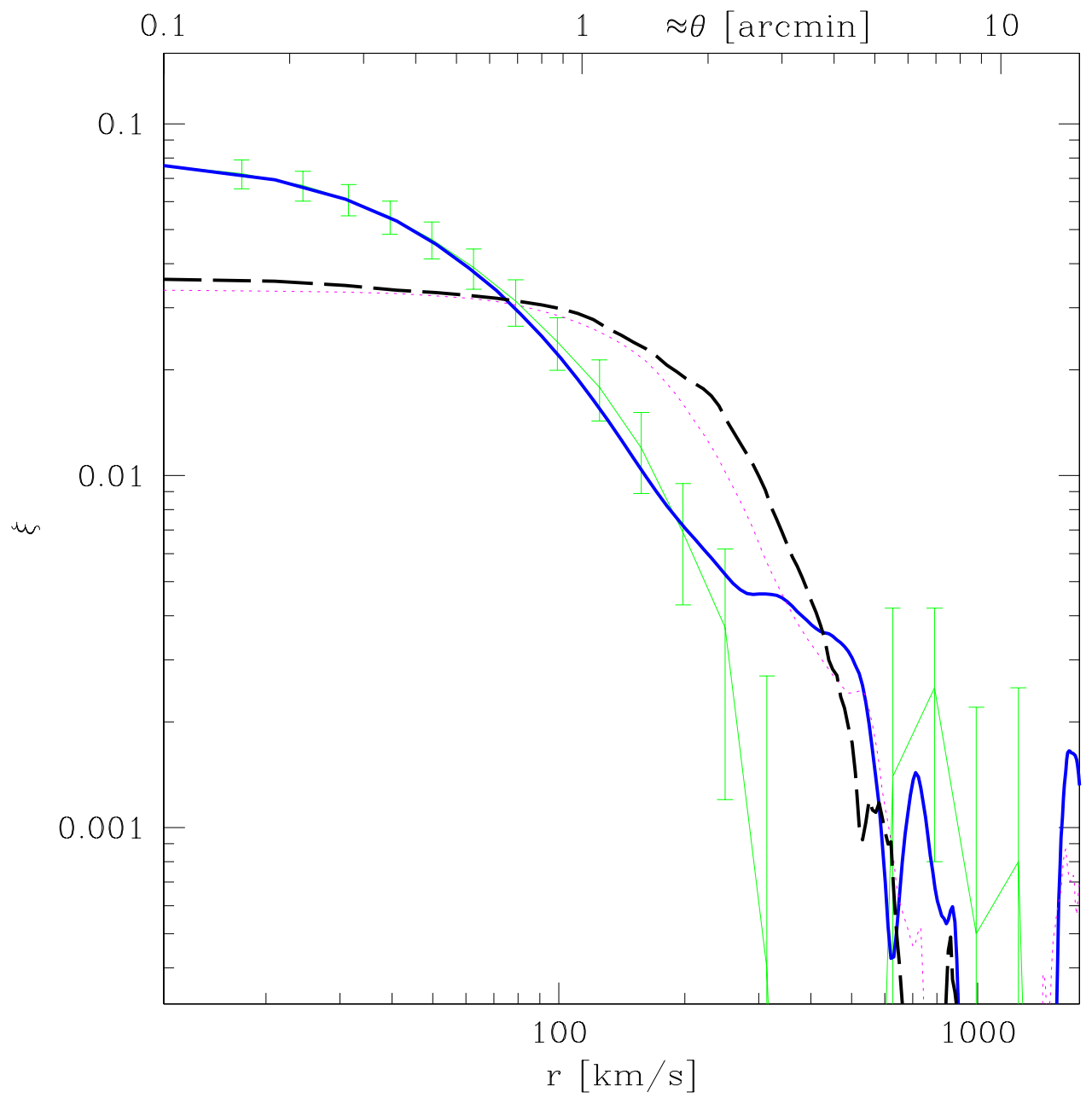

Figure 3. The longitudinal correlation function determined from the Large Programme data (thick, solid line) is in very good agreement with the findings of McDonald et al. (2000, thin solid line) using Keck data. The function derived from the high resolution data convolved with a Gaussian filter with FWHM $=220 \mathrm{~km} \mathrm{~s}^{-1}$ (thin dotted line) is consistent with the function derived from the 58 lines of sight observed with FORS (thick, dashed line).

\subsection{The observed longitudinal correlation function}

Each individual low resolution spectrum (58 in our sample) yields one estimate of the longitudinal correlation function. We combine these measurements computing the median correlation value at each velocity separation.

We also calculated the longitudinal correlation function at higher spectral resolution using the Large Programme data. For each line of sight the mean flux was calculated from all pixels in the Lyman- $\alpha$ forest along this line of sight. The corresponding correlation function shown in Fig. 3 has a mean redshift of 2.39 and is in remarkable agreement with the findings of McDonald et al. (2000) from a sample of eight lines of sight observed with Keck at a mean redshift of 2.41. As the FORS data are of much lower resolution, we have calculated the correlation function after convolving the high resolution data with 


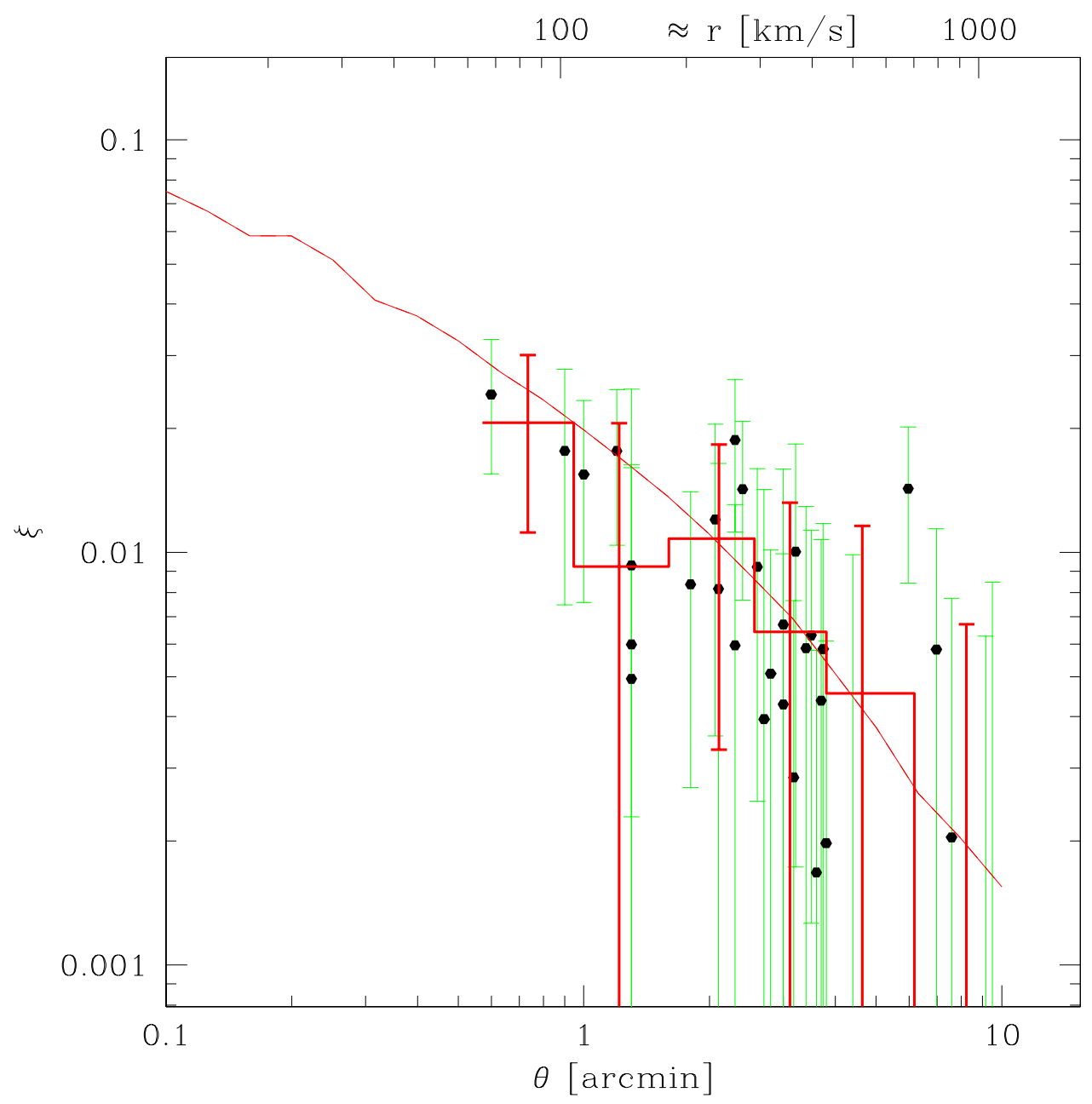

Figure 4. The observed transverse correlation coefficients (black dots and histogram) are plotted over the transverse correlation function from the hydro-simulation. In the latter case, the normalisation of the solid curve has been fixed on the longitudinal correlation function. The error bars on each black dot are computed from the pure dark-matter simulation.

a Gaussian filter of FWHM $=220 \mathrm{~km} \mathrm{~s}^{-1}$ matching the FORS resolution. The result is compared with the correlation function measured from the 58 lines of sight observed with FORS. It can be seen in Fig. 3 that the correlation functions agree very well although the low-resolution (FORS) correlation function is somewhat higher than the one from the convolved high resolution data.

\subsection{Observed transverse correlation function}

The observed transverse correlation coefficients measured from the 33 pairs are shown in Fig. 4. Over-plotted as a continuous line is the simulated correlation function measured in the hydro simulation at $z=2$. We use the $z=2$ function because the mean redshift of the Lyman- $\alpha$ forest in the pairs is $\sim 2.1$. The normalisation has been fixed by adjusting the PDF of the longitudinal correlation function. Therefore there is no adjustment of the simulated function in this plot. We have gathered the observed measurements in 
bins weighting the points by the errors. It can be seen that the agreement is quite good especially between the shapes of the observed and simulated functions. This demonstrates that the data are consistent with the current scheme describing the Lyman- $\alpha$ forest as the tracer of a continuous photoionised and warm intergalactic medium associated with the filamentary and sheet-like structures predicted in cold dark matter-like models of structure formation. However, it is apparent from the figure that although the data set is unique, it is not sufficient to significantly constrain the geometry of the Universe. This confirms the predictions of McDonald (2003) that significant constraints can be obtained on $\Omega_{\Lambda}$ with a number of pairs of the order of $13\left(\theta / 1^{\prime}\right)^{2}$ on large scales. However, our result demonstrates the presence of a strong signal in the transverse correlation on scales $<5$ arc-min and justifies the future investment in observing time that will be needed to extract information on $\Omega_{\Lambda}$.

\section{Acknowledgements}

I would like to thank our collaborators for allowing us to refer to common works: B. Aracil, R. Carswell, F. Coppolani, M. Haehnelt, C. Ledoux, M. Longhetti, C. Pichon, E. Rollinde, E. Scannapieco, R. Srianand, F. Stoehr. Data presented here was obtained at the ESO-VLT observatory on Mount Paranal, Chile.

\section{References}

Adelberger, K. L., et al., 2003, ApJ, 584, 45

Alcock, C., \& Paczyński, B., 1979, Nature, 281, 358

Aracil, B., Petitjean, P., Smette, A., et al., 2001, A\&A, 391, 1

Aracil, B., Petitjean, P., et al., 2004, A\&A, 419, 811

Bergeron, J., \& Boissé, P., 1991, A\&A, 328, 499

Churchill, C. W., et al., 2000, ApJ, 543, 577

Crotts, A. P. S., Fang, Y. H., 1998, ApJ, 502, 16

Heckman, T. M., et al., 2000, ApJS, 129, 493

McDonald, P., 2003, ApJ, 585, 34

McDonald, P., Mirada-Escudé, J., Rauch, M., et al., 2000, ApJ, 543, 1

Mac Low, M. M., Ferrara, A., 1999, ApJ, 513, 142

Petitjean, P., \& Bergeron, J., 1990, A\&A, 231, 309

Petitjean, P., Surdej, J., Smette, A., et al., 1998, A\&A, 334, L45

Rigby, J. R., et al., 2002, ApJ, 565, 743

Rollinde, E., Petitjean, P., Pichon, C., et al., 2003, MNRAS, 341, 1299

Scannapiedo, E., Pichon, C., Aracil., B., et al., 2005, astro-ph/0503001

Smette, A., et al., 1995, A\&AS, 113, 199

Schaye, J., et al., 2003, ApJ, 596, 768

Steidel, C., Sargent, W. L. W., 1992, ApJS, 80, 1

Steidel, C., et al., 1997, ApJ, 480, 568

Young, P. A., Impey, C. D., Foltz, C. B., 2001, ApJ, 549, 76 